Review

\title{
Progress on Complications of Direct Bypass for Moyamoya Disease
}

\author{
Jinlu Yu, Lei Shi, Yunbao Guo, Baofeng Xu, Kan Xu凶 \\ Department of Neurosurgery, First Hospital of Jilin University, Changchun, 130021, P.R. China \\ $\triangle$ Corresponding author: Kan Xu, Department of Neurosurgery, First Hospital of Jilin University, 71 Xinmin Avenue, Changchun 130021, China. Email: \\ jlyu@jlu.edu.cn
}

(C) Ivyspring International Publisher. Reproduction is permitted for personal, noncommercial use, provided that the article is in whole, unmodified, and properly cited. See http://ivyspring.com/terms for terms and conditions.

Received: 2016.02.26; Accepted: 2016.06.20; Published: 2016.07.05

\begin{abstract}
Moyamoya disease (MMD) involves progressive occlusion of the intracranial internal carotid artery resulting in formation of moyamoya-like vessels at the base of the brain. It can be characterized by hemorrhage or ischemia. Direct vascular bypass is the main and most effective treatment of MMD. However, patients with MMD differ from those with normal cerebral vessels. MMD patients have unstable intracranial artery hemodynamics and a poor blood flow reserve; therefore, during the direct bypass of superficial temporal artery (STA)-middle cerebral artery (MCA) anastomosis, perioperative risk factors and anesthesia can affect the hemodynamics of these patients. When brain tissue cannot tolerate a high blood flow rate, it becomes prone to hyperperfusion syndrome, which leads to neurological function defects and can even cause intracranial hemorrhage in severe cases. The brain tissue is prone to infarction when hemodynamic equilibrium is affected. In addition, bypass vessels become susceptible to occlusion or atrophy when blood resistance increases. Even compression of the temporalis affects bypass vessels. Because the STA is used in MMD surgery, the scalp becomes ischemic and is likely to develop necrosis and infection. These complications of MMD surgery are difficult to manage and are not well understood. To date, no systematic studies of the complications that occur after direct bypass in MMD have been performed, and reported complications are hidden among various case studies; therefore, this paper presents a review and summary of the literature in PubMed on the complications of direct bypass in MMD.
\end{abstract}

Key words: moyamoya disease, direct bypass, complications, progress

\section{Introduction}

Moyamoya disease (MMD) involves progressive stenosis of the bilateral intracranial internal carotid arteries at their ends and formation of circuitous and expanded moyamoya-like vessels at the base of the brain [1]. MMD occurs in both children and adults and is mainly characterized by ischemic or hemorrhagic stroke. It often requires treatment to prevent stroke. Treatments of MMD include direct and indirect bypasses, and superficial temporal artery (STA)-middle cerebral artery (MCA) bypass is the main direct bypass surgery performed [2]. Direct bypass can be equally effective in preventing ischemic stroke in both adults and children with ischemia [3-5].
Regarding hemorrhagic MMD, direct bypass surgery potentially decreases hemodynamic stress on moyamoya vessels [6]. In addition, it restores damaged blood vessels and plays a role in preventing hemorrhagic stroke [7]. Whether direct STA-MCA bypass reduces the likelihood of MMD rehemorrhage is controversial, and most studies have suggested that it does not reduce rehemorrhage [8-11]. Further, studies have suggested that combined external carotid artery (ECA)- internal carotid artery (ICA) bypass may be more beneficial than conservative therapies for hemorrhagic MMD patients and that improvements in dilation and branch extension of the 
anterior choroidal artery (AchA)- posterior communicating artery (PcoA) after this operation might be correlated with a lower rehemorrhage rate $[12,13]$. Therefore, direct bypass is the most important and effective treatment of MMD.

MMD is pathophysiologically complex. Fragile MMD vessels and unstable hemodynamics result in a very high surgical risk, and direct bypass is associated with a high incidence of surgical complications, especially in patients with unstable MMD $[14,15]$. In 2005, Kim et al. found that MMD surgery performed within 6 weeks after the last ischemic infarction resulted in a higher incidence of postoperative ischemic complications [16]. However, direct bypass is associated with a much lower surgical risk in stable MMD, which is characterized by headaches. In 2012, Okada et al. used direct bypass to treat 25 patients with ischemic MMD suffering from severe headaches and observed no complications after the operations were performed [17].

The exact incidence of MMD complications occurring after direct bypass is still unknown. In 2009, Guzman et al. evaluated the effects of 450 revascularization procedures performed on MMD patients on their clinical outcomes; they found that the surgical morbidity rate was $3.5 \%$ and that the mortality rate was $0.7 \%$ per treated hemisphere [18]. The main causes of MMD complications are hemodynamic fluctuations caused by bypass surgery, as well as other types of surgery and anesthesia [15]. Complications mainly include hyperperfusion syndrome, postoperative cerebral hemorrhage, postoperative cerebral infarction, vascular bypass occlusion, bypass anastomotic aneurysm, and scalp necrosis, among others.

No systematic studies have been conducted to assess the complications that occur after direct bypass in MMD, and the reported complications are hidden among various case studies; therefore, this paper presents a review and summary of the literature in PubMed regarding the complications that occur after direct bypass in MMD.

\section{Hyperperfusion syndrome (HS)-induced local neurological impairment}

This complication has gradually been recognized in recent years, and it is the most common complication of direct bypass in MMD. Symptomatic HS is defined as the presence of a significant increase in cerebral blood folw (CBF) at the site of anastomosis that is responsible for the apparent neurological signs [19].

Direct STA-MCA surgery for MMD used to be considered as low-flow anastomosis with little possibility of HS; in fact, the incidence of HS after direct bypass in MMD is not low [20]. Fujimura et al. reported that among 80 hemisphere surgeries of 50 MMD patients, the incidence of HS was $27.5 \%$ (22/80)[19]. This group updated their research in 2011 and found that among 121 hemisphere surgeries of 80 MMD patients, the incidence of HS was $21.5 \%$ (26/121)[21]. They updated their data again in 2012, reporting that among 150 hemisphere surgeries of 106 consecutive MMD patients, the incidence of HS was $18 \%(27 / 150)$ [22]. In 2012, Uchino et al. found that radiological hyperperfusion occurred in $50 \%$ of MMD patients after surgery, and this rate was higher than previously thought. Notably, adult patients are at a much higher risk of postoperative hyperperfusion than pediatric patients [23].

\section{The pathogenetic mechanism of HS}

The pathogenetic mechanism of HS after direct bypass in MMD is similar to that of HS after surgery for internal carotid artery stenosis. Both are symptoms that appear after a sudden increase in blood flow in arteries of the brain with impaired automatic adjustment due to long-term ischemia [24]. Although STA-MCA surgery for the treatment of MMD is a low-flow surgery, HS may occur in reperfused areas of the cerebral cortex because the thickened STA might not match with the diameter of the atrophic cerebral cortex artery, i.e., the blood flow is lower in the reconstructed cerebral cortex artery. This phenomenon has been observed using imaging and assessments of blood flow dynamics [25, 26]. MMD direct bypass has also been reported to cause dynamic changes in postoperative cerebral hemodynamics with the occurrence of watershed shift and hyperperfusion, leading to postoperative neurological deterioration [27]. Further, delayed pseudolaminar necrosis can be observed in the resulting damaged cortex via imaging [28].

In addition to the impaired automatic adjustment of cerebral vessels, other mechanisms are involved in the occurrence of HS. Studies have found that MMD direct bypass with reperfusion results in generation of a large amount of oxygen free radicals, which promotes the development of HS or aggravates HS by further injuring damaged vessels [29, 30]. Moreover, the cerebral cortices of patients with MMD and long-term ischemia may exhibit excessively high expression levels of angiogenesis factors and extracellular matrix proteins, affecting blood vessel permeability and thus inducing HS [31, 32]. Inflammation may also be involved in the occurrence of HS. In 2013, Hwang et al. found that in MMD direct bypass, an increased postoperative WBC count was associated with postoperative, transient neurological deterioration due to cerebral hyperperfusion after 
STA-MCA anastomosis in adult MMD patients [33].

The occurrence of HS after MMD direct bypass is associated with blood flow from the STA, and research has been performed to investigate whether it is also associated with double bypass with 2 STAs. In 1995, Takahashi et al. suggested that vascular reconstruction centered on the frontal region utilizing both the anterior and posterior branches of the STA was more efficacious[34]. However, other studies have suggested that single bypass is sufficient for the treatment of MMD and that it is associated with a lower risk of HS [12, 35, 36].

\section{Clinical manifestation of HS}

The clinical symptoms of HS can be divided into two categories, namely focal HS with transient neurological deficits and intracranial hemorrhage caused by HS. The latter is a serious complication that is discussed in the next section. Local HS complications can present as headaches and partial neurological deficits. The first definite case of HS caused by STA-MCA revascularization in the treatment of MMD was reported by Uno in 1998. A 47-year-old woman presented with hemiplegia and aphasia on the second day after surgery. MRI was performed, revealing edema in the blood supply area, and SPECT suggested increased local blood flow, which was determined to be HS. The symptoms gradually subsided over 17 days after onset, and they ultimately disappeared [37]. Symptoms caused by HS are more prevalent than hemisphere symptoms, but in rare cases, transient crossed cerebellar diaschisis may occur due to cerebral hyperperfusion following surgical revascularization in MMD [38].

It has been widely accepted that HS is caused by vascular reconstruction in the treatment of MMD [26]. Most of the symptoms of HS caused by MMD direct bypass are relieved after surgery. In 2011, Hayashi et al. carried out retrospective analysis of $5 \mathrm{MMD}$ patients who developed HS after vascular reconstruction, and they found that the HS symptoms generally appeared during the first postoperative week and were relieved during the second postoperative week [39]. However, in some cases, HS continued until the fifth postoperative week [40]. Notably, if HS causes neural damage, then permanent sequelae can manifest [30, 41].

\section{Imaging detection of HS}

Methods used for detection of HS include computed tomography (CT), magnetic resonance imaging (MRI), transcranial cerebral doppler) (TCD), and single photon emission computed tomography (SPECT), among others [42]. CT and MRI are morphological detection methods that can be used to identify indirect evidence of HS, such as hemorrhage and edema [43]. TCD and SPECT can be applied to dynamically detect hemodynamic changes around the anastomotic stoma [44, 45]. SPECT is the gold standard method for detecting intracranial vascular blood flow before and after reconstruction, and it is often carried out within 48-72 hours after surgery for the early detection of HS [46].

In addition, perfusion CT and MRI are alternative methods used to detect increased blood flow in anastomotic areas $[47,48]$. Some inspection methods also have certain significance in the prediction of HS. In 2015, Noguchi et al. used a noninvasive method, ASL-MRI, to map cerebrovascular resistance in MMD and found that it was useful for detecting the occurrence of HS [24]. In 2011, Kawamata et al. used laser doppler to examine cerebral blood flow in MMD patients before and after reconstruction to predict the occurrence of HS; their results suggested that HS might develop when the blood flow rate is increased by over 100\% [49]. Moreover, in 2009, Nakagawa et al. predicted the occurrence of HS by monitoring the temperature of surrounding brain tissue with infrared rays. They found that after vascular reconstruction, if the temperature of the surrounding anastomotic brain tissue was increased by a certain amount, then HS would occur [50]. In 2014, Machida et al. found that intraoperative venous reddening could also serve as a possible sign of hyperperfusion [51]. In 2015, Fujimura et al. emphasized that quantitative analysis of early postoperative $\mathrm{CBF}$ was useful for predicting and diagnosing HS after MMD revascularization surgery [48].

\section{Treatments of HS}

The functional loss of cerebrovascular regulation due to long-term ischemia causes HS after STA-MCA bypass in MMD; therefore, the most direct HS treatment is to control blood pressure $[19,52]$. Uno et al. have suggested that blood pressure should be maintained within the normal range for the treatment of HS, with a systolic blood pressure of 120-140 $\mathrm{mmHg}$ [37]. However, Ogasawara have suggested that systolic blood pressure should be maintained at 90-120 mmHg [41].

Because large amounts of oxygen free radicals, which are considered directly related to the occurrence of HS, are produced when cerebral reperfusion occurs after MMD direct bypass, an oxygen free radical scavenger such as edaravone can be administered for treatment [41]. Edema may occur in brain tissues affected by HS, and it can be treated with dehydrating agents [53]. Most cases of HS that occur after MMD direct bypass are reversible, and 
satisfactory outcomes can be achieved after the appropriate treatment is administered; the symptoms of HS are often relieved within 2 weeks [54].

Minocycline is a neuroprotective antibiotic agent that blocks matrix metalloproteinase-9, which contributes to edema formation and hemorrhagic conversion after cerebral ischemia-reperfusion. In 2014, Fujimura et al. studied the use of minocycline to treat MMD patients with HS and found that it effectively controlled this complication after direct bypass [55].

\section{HS-induced intracranial hemorrhage}

In addition to headaches and local neurofunctional deficits, the most serious consequence of HS is intracranial hemorrhage [56]. The occurrence of HS-induced intracerebral hemorrhage is not rare after direct revascularization of MMD. In 2009, Guzman et al. found that after performing 450 revascularization procedures on MMD patients, postoperative bleeding occurred in 8 patients. All new hemorrhages occurred in previously ischemic territories, and they were all in the surgical hemisphere and were caused by HS, with an incidence of $1.7 \%(8 / 450)$ [18]. In 2014, Kazumata et al. found that the frequency of postoperative stroke was $1.3 \%$ in MMD patients following combined revascularization and the appearance of hemorrhagic complications [57].

\section{Characteristics of hemorrhage}

HS-induced hemorrhage includes intracranial hematoma and subarachnoid hemorrhage. CT indicators of postoperative hemorrhage are compression or displacement of the brain, even if they are minimal. In 2009, Fujimura et al. reported on a 47-year-old woman whose postoperative MRI and SPECT results showed increased blood flow in the anastomosis blood supply area, indicative of vasogenic cerebral edema and HS. The patient presented with a sudden severe headache and monoplegia of the upper limbs on postoperative day 4, and head CT showed intracranial hematoma [53]. In addition, Fujimura reported on a group of 13 HS patients, among which arachnoid hemorrhage occurred in 2 [58].

\section{Hemorrhage occurrence time}

In 2014, Schubert et al. found that HS-induced hemorrhage often occurred within 7 days after MMD direct bypass [59]. However, Guzman studied 8 cases of MMD patients who received direct bypass and found that hemorrhage occurred during surgery in one patient, while it occurred between 4 hours and 10 days after surgery in the other patients [18]. In 2011,
Ito et al. reported on 1 patient with hemorrhage after MMD direct bypass who exhibited cerebral hemorrhage due to HS at 7 days after surgery [60]. In 2014, Kazumata et al. found that most cases of postoperative hemorrhage occurred following combined revascularization for MMD either during surgery or within 4 weeks after surgery [57]. Based on these observations, hemorrhage could occur anytime, from the intraoperative period to 1 month after surgery.

\section{Prognosis}

HS prognosis mainly depends on the amount of blood loss. Fujimura et al. reported on 1 patient who developed HS-induced intracranial hematoma after MMD direct bypass and exhibited good recovery after conservative treatment due to a small amount of blood loss [53]. Ideally, no clinical symptoms should remain after recovery from HS-induced hemorrhage. In 2011, Ito reported one patient with HS without asymptomatic hemorrhage [60]. Notably, if a hemorrhage leads to extensive blood loss, then prognosis is very poor. Mesiwala et al. carried out retrospective analysis of 39 MMD patients who underwent direct revascularization of 65 hemispheres; fatal HS-induced intracranial hemorrhage occurred in 2 of the patients during the immediate postoperative period far from the surgical site [61]. Guzman et al. included 8 patients with postoperative hemorrhage in their study conducted in 2009. Only 5 of the patients completely recovered, 2 died, and 1 developed hemiplegia and aphasia [18].

\section{Postoperative infarction and non-HS-induced hemorrhage}

Postoperative infarction and non-HS-induced hemorrhage have similar mechanisms and prognoses. These postoperative complications are closely related to the hemodynamic instability characteristic of MMD. Unilateral direct bypass in symptomatic hemispheres of MMD patients could cause impairment of cerebrovascular regulation in primary asymptomatic hemispheres shortly after surgery, which may result in the occurrence of infarction and hemorrhage in the whole brain [62, 63]. Even anesthesia can induce intraoperative, acute brain swellin [64].

\section{Postoperative infarction}

Postoperative ischemia after MMD direct bypass refers to any new hypodensity detected on $\mathrm{CT}$, and it also includes lacunar infarction [59]. Infarction after MMD direct bypass is not uncommon. Kazumata et al. enrolled 236 patients who underwent 358 revascularization procedures in their single-center, 
multiple-case study conducted in 2014, and they found that the frequency of postoperative ischemic stroke in MMD following combined direct and indirect revascularization was $4.1 \%$ [57], which is in accordance with the results of other studies. In 2011, Jung et al. found that among 79 hemispheres of 73 consecutive patients with adult MMD who underwent direct bypass surgery, ischemic complications occurred in $11.4 \%$ (9 of 79), many of which could be attributed to advanced MMD, such as Suzuki stages 3 and 4 [65].

\section{Causes}

MMD postoperative ischemic complications are closely related to hemodynamic instability. Many studies have found that several factors, such as hypercapnia, hypotension and inadequate hematocrit, increase the risk of postoperative ischemia [66, 67]. In 1997, Sato et al. have identified 3 specific risk factors, including the presence of a preoperative low-density area, intraoperative urinary output, and reduced hematocrit [68]. In 2010, Hyun et al. emphasized that hypercapnia was an important contributor to MMD [69]. However, the intraoperative intracerebral steal phenomenon has also been found to be associated with global hyperemia in MMD [70]. These risk factors are associated with anesthesia and perioperative risk [71].

Ischemic complications often occur in the surgical hemisphere, but they can also occur in the contralateral hemisphere. In 2009, Guzman et al. reported on 8 MMD patients who developed combined ischemia after direct bypass, and they found that stroke occurred in the surgical hemisphere in 4 patients and on the contralateral side in 4 [18]. In addition, early graft occlusion in an unblocked bypass can lead to cerebral ischemia [3]. In conclusion, acute preoperative infarcts and poor cerebrovascular reserve are independent risk factors for severe ischemic complications following direct extracranialintracranial bypass in MMD [72].

\section{Infarction occurrence time}

The occurrence time of infarction after MMD direct bypass remains unclear. In 2000, Kuroda et al. reported on 2 patients with frontal infarction that developed at 3-4 days after surgery [73]. In addition, Hyun et al. reported on 2 patients who developed infarction after MMD direct bypass and found that the infarctions occurred within $24 \mathrm{~h}$ after surgery in both patients [69]. In 2014, Schubert et al. have found that most ischemic events occur late after MMD direct bypass, with a particularly high frequency of events occurring within 7 days after surgery [59]. Therefore, postoperative infarction can occur within the first postoperative day, but it has a high incidence within the first postoperative week.

\section{Prognosis}

The prognosis of infarction after MMD direct bypass depends on its location and severity. Some infarctions are silent and do not cause clinical symptoms. In a study conducted by Mesiwala et al. in 2008, 5 patients developed silent infarcts. These complications were relatively minor and were detected using postoperative diffusion-weighted magnetic resonance imaging [61]. However, the occurrence of a silent infarction also promotes the development of unstable MMD, which should be closely monitored; otherwise, it may potentially cause ischemic or hemorrhagic stroke [74]. Some infarctions can be fatal. In 2008, Guzman et al. reported on 8 patients who had suffered from a new stroke, of whom only 4 completely recovered, 1 died, and the remaining 3 developed sequelae [18].

\section{Prevention}

Hemodynamics should be kept stable after MMD direct bypass to prevent the occurrence of postoperative infarction. However, some studies have examined other methods for preventing their occurrence. Schubert et al. advocated antiplatelet therapy in 2014, reporting that it did not increase the risk of hemorrhagic complications and that it may improve outcome [59]. In contrast, there have also been reports that do not support antiplatelet therapy. In 2016, Yamada et al. found that the rates of cerebral infarction did not significantly differ between an ischemic group and antiplatelet and non-antiplatelet subgroups [75].

\section{Non-HS-induced intracranial hemorrhage}

Most postoperative cerebral hemorrhages occurring after MMD direct bypass are caused by excessive perfusion, while some are caused by hemodynamic changes. It is difficult to identify causes in such cases. However, contralateral cerebral hemorrhage is not considered to be caused by hyperperfusion. This type of hemorrhage is speculated to be associated with perioperative hemodynamic changes and contralateral vascular rupture hemorrhage induced by anesthesia. Januszewski et al. reported on 1 MMD patient with collateral hemorrhage that appeared on the third day after STA-MCA treatment in 2014 [3]. Therefore, the stability of perioperative hemodynamics in MMD patients should be considered. 


\section{Complications of bypass graft vessels}

\section{Postoperative bypass occlusion}

STA-MCA bypass grafts can be occluded after MMD direct bypass. This occlusion is associated with many factors; aside from anastomosis, the main reason is the higher flow resistance of the bypass.

\section{Bypass hemodynamics}

In 2014, Kazumata et al. classified STA-MCA bypass using near-infrared indocyanine green video angiography technology into the following three types: type I, which is the most common and is characterized by robust anterograde flow during the early arterial phase; type II, characterized by delayed but patent graft enhancement and anterograde flow during the capillary or venous phase; and type III, characterized by delayed anterograde flow, with no continuity to the bypass site or with no convincing flow at all. They also assessed whether the classification carried any significance in the prediction of bypass occlusion. Among 36 procedures performed, this group detected bypass occlusion in 4 , all of which occurred in patients with poor blood flow, including 1 with type II and 3 with type III [56].

These occurrences were thought to be caused by direct competition of the recipient vessel with the bypass for regional cerebral blood flow, with no demand of the bypass for input blood with no demand of the bypass. Iwama et al. found one patient who underwent simultaneous STA-anterior cerebral artery (ACA) and STA-MCA anastomoses and had poor bypass flow in their study conducted in 1998. This was probably caused by formation of spontaneous leptomeningeal collateral channels between the ACA and MCA [76].

\section{Occurrence time}

The reported times of bypass occlusion have varied. In Kazumata's study, most bypass occlusions occurred within 24-72 hours after surgery [56]. In Abla's study conducted in 2013, among 154 revascularization surgeries on 140 affected hemispheres, 4 developed bypass occlusion after surgery, one of which occurred immediately after surgery, and the other 3 of which developed occlusion at 9,33, and 64 months after surgery, respectively [3]. Therefore, the time of occurrence of bypass occlusion is uncertain, but it is mainly related to distal resistance of the bypass.

\section{Treatment}

Once bypass occlusion occurs, in the absence of sufficient collateral circulation or combined indirect revascularization, surgical revision may be required after the initial procedure is performed. In Abla's study conducted in 2013, among 4 patients with MMD complications, 2 continued underwent bypass surgery, while the other 2 were conservatively treated according to their conditions [3]. In Freyschlag's study performed in 2011, direct extra-intracranial bypass surgery in combination with encephalo-myosynangiosis was performed on 71 MMD patients, including 2 with complete occlusion of the direct anastomosis [77]. However, those patients were not treated because no clinical symptoms were present.

Bypass occlusion is associated with many factors. Perhaps it should not be called a complication, but it still needs to be mentioned here. Amin-Hanjani carried out a study of combined direct and indirect bypass for MMD about quantitative assessment of iirect bypass flow over time in 2013 and found a frequently reciprocal relationship between direct STA bypass flow and indirect encephalo-duro-arteriosynangiosis (EDAS) collaterals. When indirect bypass predominates, it can gradually shrink to the point of occlusion [78].

\section{Reversible occlusion}

During MMD STA-MCA surgery, because the STA needs to penetrate through the temporalis to be anastomosed with the MCA of the cerebral cortex, the activities of the temporalis may cause the STA to promote the development of temporary ischemia in the cerebral cortex upon widely opening the mouth. In 2015, Katsuta et al. reported on 5 adult MMD patients who developed ischemia upon wide mouth opening. Their study revealed the presence of at least the following 2 steno-occlusive mechanisms: (1) the stretched temporalis muscle fibers push the donor STA against the edge of the bone window; and (2)the redundant donor STA kinks when the muscle is stretched. To avoid this phenomenon, it is recommended that a sufficient distance is secured between the donor STA and the edge of the bone window and that a redundant course of the donor STA within the muscle layer is avoided [79].

Bypass ischemia that occurs due to opening of the mouth is not rare. As early as 2011, Freyschlag et al. reported on $71 \mathrm{MMD}$ patients who underwent direct extra-intracranial bypass surgery in combination with encephalomyosynangiosis, 3 of whom exhibited alterations in bypass patency upon mouth opening [77].

\section{Bypass graft spasm}

Bypass vessels pass subcutaneously under the cheeks and are easily affected by the temporalis, so they are prone to vasospasms. Bypass vasospasms have been reported to occur in the treatment of 
intracranial aneurysms [80]. Bypass spasms are relatively rare in MMD but have been reported. Natarajan et al. described one MMD patient who developed radial vasospasms after direct bypass with radial artery reconstruction in 2010. Over-the-wire balloon angioplasty was performed, and a nitroglycerin patch was applied cutaneously over the graft to relieve the vasospasms [81]. Thus, the possibility of this rare complication should also be considered.

\section{Anastomotic aneurysm}

STA-MCA anastomosis is an effective treatment of MMD-related ischemia [82]. End-to-side anastomosis of the STA and MCA is necessary for vascular reconstruction. The sutured artery is prone to forming aneurysms due to long-term impacts; moreover, anastomotic aneurysms can also occur in the distal end [83].

Treatment is needed if the aneurysm ruptures. In 2005, Nishimoto reported on 1 MMD patient who had been treated with anastomoses of the STA and MCA but developed an anastomotic aneurysm 20 years later. The patient was treated with surgical clipping; the parent artery remained, and the prognosis was good [84]. Eom et al. reported on one patient with a large bypass anastomotic aneurysm in 2010. The aneurysm caused formation of a brain hematoma after it ruptured and hemorrhaged. The hematoma was removed, and then the aneurysm was excised, but the patient was left with postoperative limb hemiplegia [85].

This type of aneurysm is caused by increased hemodynamic pressure. Cases similar to MMD bypass have also been reported. In 2013, Kawahara reported on one patient with lateral internal carotid artery occlusion. The patient was treated with STA and MCA anastomosis, and an intracranial hemorrhage developed 22 months later, forming a bypass anastomotic aneurysm. Aneurysm clipping was performed to preserve the parent artery, and the patient had a good prognosis [86]. In 2015, Yu et al. published a literature review on MMD and aneurysms. They suggested that increased hemodynamic pressure was the factor causing anastomotic aneurysm. Therefore, the possibility of anastomotic aneurysm should be considered during the follow-up of MMD patients [87].

\section{Local hypoperfusion}

The cortex arteries should be blocked temporarily during STA-MCA bypass, which results in local hypoperfusion of the cerebral cortex. Clinical symptoms often occur after surgery due to the poor compensatory ability of MMD vessels. In 2015,
Mukerji et al. studied postoperative cerebral perfusion in 31 MMD patients who underwent direct ECA-ICA bypass in 2015 and suggested that hyperperfusion was the cause of transient neurological events [88]. Other reports in the literature have also found that temporary vessel occlusion during standard ECA-ICA bypass surgery is a risk factor for intraoperative ischemia [89].

In addition, increased blood flow is achieved only in a limited area around the surgical field in most MMD patients after direct bypass surgery. Due to the influences of surgery and anesthesia on the surgical hemisphere and the characteristics of MMD, CBF images show a low-perfusion state with a non-infarcted ischemic pattern in $71 \%$ of revascularized hemispheres, especially in the frontal lobe, which results in a chronic low-perfusion state [90].

\section{Poor scalp healing and infection}

MMD direct bypass requires separation of the STA from the scalp, which destroys the blood supply to the scalp; therefore, the scalp is prone to poor healing and infection. In particular, revascularization surgery seems to be associated with a higher risk of wound-related complications. Double procedures, which use both branches of the STA, and a history of diabetes have been found to be risk factors for wound-related complications [91].

There are few reports of scalp complications after MMD bypass. Mesiwala reported on 65 total procedures performed for the treatment of MMD. They found that STA-MCA bypass was technically possible in 56 procedures and that 3 patients developed wound infection [61]. Abla et al. studied 153 revascularization surgeries in 140 affected hemispheres and found that 1 patient required reoperation due to scalp infection [3]. Houkin et al. studied direct and indirect revascularization in the context of MMD surgical techniques and perioperative complications and found that among 112 surgically treated hemispheres, skin necrosis along the skin incision occurred in 2 [92].

Scalp necrosis and infection are difficult to treat. Because scalp vascularity is severely compromised after MMD surgery, reconstruction of defects with local scalp tissue is challenging. To cover defects, a highly vascularized flap with excellent mobility and coverage is required to avoid existing scars. Kwon et al. reported on postoperative scalp necrosis in 5 patients who underwent STA-MCA anastomosis using encephalo-duro-arterio-myo-synangiosis (EDAMS). The ipsilateral occipital artery was traced, and an advancement flap based on the occipital artery and vein was designed to fit the defect. This approach 
achieved satisfactory results [93].

\section{Other complications}

In addition to the complications mentioned above, other complications have been associated with the increased blood flow caused by MMD direct bypass.

\section{Postoperative epilepsy}

The incidence of postoperative epilepsy is not low after MMD direct bypass. In 2011, Jin et al. found that the overall incidence of seizures after revascularization in adult MMD patients was $18.9 \%$, which was not lower than the incidences of other pathologies, and that the occurrence of postoperative epilepsy was mainly related to synangiosis. Early epilepsy is associated with increased bypass blood flow. Advanced epilepsy, in addition to being associated with the increased cerebral cortex excitability caused by increased blood flow, is also associated with combined indirect bypass surgery; the better the blood flow is reconstructed, the greater the risk of seizures. MMD patients with delayed postoperative seizures have also been shown to have good outcomes following direct anastomosis and synangiosis [94].

Epilepsy can also occur in HS. In 2007, Narisawa et al. performed STA-MCA anastomosis on 64 sides of 44 consecutive patients, of whom 3 suffered from seizures at between 1 and 10 days after surgery. STA-MCA anastomosis can cause hyperperfusion in patientsSTA-MCA anastomosis can be caused by hyperperfusion in patients [95]. Therefore, in contrast with previous understanding, the likelihood of postoperative epilepsy after MMD direct bypass should be considered.

\section{Subdural hematoma}

Some with MMD patients with brain atrophy and others with the long-term use of anticoagulation medication for ischemia are prone to subdural hematoma after bypass surgery. Andoh et al. reported on 3 patients who developed postoperative chronic subdural hematoma after STA-MCA bypass for MMD in 1992; this group suggested that chronic subdural hematoma may occur in patients with pre-existing brain atrophy and postoperative subdural effusion. Anticoagulant therapy should be avoided during the early postoperative period after bypass surgery [96].

\section{Transient cheiro-oral syndrome (COS)}

Transient COS refers to sensory disturbances around the corner of the mouth and in the hand without motor impairment; it is rarely observed after MMD surgery. Sasamori et al. studied surgical revascularization for MMD in 2010 and found that transient COS developed in $8(22.9 \%)$ of 35 treated hemispheres. Bypass flow through the STA-MCA anastomosis may stimulate rapid disease progression and diminish basal moyamoya vessels, causing transient COS within 3 weeks after surgery [97].

\section{Arteriovenous fistula (AVF)}

Bypass of the STA and MCA in the cortex may cause iatrogenic pial AVF. Feroze et al. reported on one patient who developed an AVF after undergoing revascularization bypass for the treatment of MMD in 2015; they have suggested that AVFs have an iatrogenic, traumatic origin and that it is possible that the incidence and progression of fistulization are influenced, in part, by intraoperative dissection and the technique used [98].

\section{Conclusion}

Various complications can occur in MMD direct bypass surgery due to the sudden increase in cerebral blood flow or hemodynamic changes caused by perioperative risk factors and anesthesia, such as HS, cerebral hemorrhage and cerebral infarction, bypass occlusion caused by distal vascular resistance, bypass occlusion caused by compression of the temporalis, and anastomotic aneurysm. These complications occur due to MMD bypass treatment, and they must be considered and avoided as much as possible.

\section{Competing Interests}

The authors have declared that no competing interest exists.

\section{References}

1. Research Committee on the P, Treatment of Spontaneous Occlusion of the Circle of W, Health Labour Sciences Research Grant for Research on Measures for Infractable D. Guidelines for diagnosis and treatment of moyamoya disease (spontaneous occlusion of the circle of Willis). Neurol Med Chir (Tokyo). 2012; 52: 245-66.

2. Kim JE, Jeon JS. An update on the diagnosis and treatment of adult Moyamoya disease taking into consideration controversial issues. Neurol Res. 2014; 36: 407-16.

3. Abla AA, Gandhoke G, Clark JC, Oppenlander ME, Velat GJ, Zabramski JM, et al. Surgical outcomes for moyamoya angiopathy at barrow neurological institute with comparison of adult indirect encephaloduroarteriosynangiosis bypass, adult direct superficial temporal artery-to-middle cerebral artery bypass, and pediatric bypass: 154 revascularization surgeries in 140 affected hemispheres. Neurosurgery. 2013; 73: 430-9.

4. Ng J, Thompson D, Lumley JP, Saunders DE, Ganesan V. Surgical revascularisation for childhood moyamoya. Childs Nerv Syst. 2012; 28: 1041-8.

5. Kim T, Oh CW, Kwon OK, Hwang G, Kim JE, Kang HS, et al. Stroke prevention by direct revascularization for patients with adult-onset moyamoya disease presenting with ischemia. J Neurosurg. 2015: 1-6.

6. Morimoto M, Iwama T, Hashimoto N, Kojima A, Hayashida K. Efficacy of direct revascularization in adult Moyamoya disease: haemodynamic evaluation by positron emission tomography. Acta Neurochir (Wien). 1999; 141: 377-84

7. Han JS, Abou-Hamden A, Mandell DM, Poublanc J, Crawley AP, Fisher JA, et al. Impact of extracranial-intracranial bypass on cerebrovascular reactivity and clinical outcome in patients with symptomatic moyamoya vasculopathy. Stroke. 2011; 42: 3047-54

8. Yoshida $Y$, Yoshimoto $T$, Shirane $R$, Sakurai $Y$. Clinical course, surgical management, and long-term outcome of moyamoya patients with rebleeding after an episode of intracerebral hemorrhage: An extensive follow-Up study. Stroke. 1999; 30: 2272-6. 
9. Aoki N. Cerebrovascular bypass surgery for the treatment of Moyamoya disease: unsatisfactory outcome in the patients presenting with intracranial hemorrhage. Surg Neurol. 1993; 40: 372-7.

10. Huang Z, Ding X, Men W, Zhang D, Zhao Y, Wang R, et al. Clinical features and outcomes in 154 patients with haemorrhagic moyamoya disease: comparison of conservative treatment and surgical revascularization. Neurol Res. 2015; 37: 886-92.

11. Okada $Y$, Shima $T$, Nishida M, Yamane $K$, Yamada $T$, Yamanaka C. Effectiveness of superficial temporal artery-middle cerebral artery anastomosis in adult moyamoya disease: cerebral hemodynamics and clinical course in ischemic and hemorrhagic varieties. Stroke. 1998; 29: 625-30.

12. Jiang H, Ni W, Xu B, Lei Y, Tian Y, Xu F, et al. Outcome in adult patients with hemorrhagic moyamoya disease after combined extracranial-intracranial bypass. J Neurosurg. 2014; 121: 1048-55.

13. Miyamoto S, Yoshimoto T, Hashimoto N, Okada Y, Tsuji I, Tominaga T, et al. Effects of extracranial-intracranial bypass for patients with hemorrhagic moyamoya disease: results of the Japan Adult Moyamoya Trial. Stroke. 2014; 45: 1415-21.

14. Haque A, Quint DJ, Castle VP, Leber SM. Another Rare Unstable Hemoglobinopathy: Hemoglobin Casper/Southampton Associated with Moyamoya Disease. Cerebrovasc Dis Extra. 2015; 5: 52-4.

15. Funaki T, Takahashi JC, Takagi Y, Kikuchi T, Yoshida K, Mitsuhara T, et al. Unstable moyamoya disease: clinical features and impact on perioperative ischemic complications. J Neurosurg. 2015; 122: 400-7.

16. Kim SH, Choi JU, Yang KH, Kim TG, Kim DS. Risk factors for postoperative ischemic complications in patients with moyamoya disease. J Neurosurg. 2005; 103: 433-8.

17. Okada Y, Kawamata T, Kawashima A, Yamaguchi K, Ono Y, Hori T. The efficacy of superficial temporal artery-middle cerebral artery anastomosis in patients with moyamoya disease complaining of severe headache. J Neurosurg. 2012; 116: 672-9.

18. Guzman R, Lee M, Achrol A, Bell-Stephens T, Kelly M, Do HM, et al. Clinical outcome after 450 revascularization procedures for moyamoya disease. Clinical article. J Neurosurg. 2009; 111: 927-35.

19. Fujimura M, Mugikura $S$, Kaneta $T$, Shimizu H, Tominaga T. Incidence and risk factors for symptomatic cerebral hyperperfusion after superficial temporal artery-middle cerebral artery anastomosis in patients with moyamoya disease. Surg Neurol. 2009; 71: 442-7.

20. Kim JE, Oh CW, Kwon OK, Park SQ, Kim SE, Kim YK. Transient hyperperfusion after superficial temporal artery/middle cerebral artery bypass surgery as a possible cause of postoperative transient neurological deterioration. Cerebrovasc Dis. 2008; 25: 580-6.

21. Fujimura $M$, Shimizu $H$, Inoue $T$, Mugikura S, Saito A, Tominaga $T$. Significance of focal cerebral hyperperfusion as a cause of transient neurologic deterioration after extracranial-intracranial bypass for moyamoya disease: comparative study with non-moyamoya patients using N-isopropyl-p-[(123)I]iodoamphetamine single-photon emission computed tomography. Neurosurgery. 2011; 68: 957-64; discussion 64-5.

22. Fujimura $M$, Tominaga $T$. Lessons learned from moyamoya disease: outcome of direct/indirect revascularization surgery for 150 affected hemispheres. Neurol Med Chir (Tokyo). 2012; 52: 327-32.

23. Uchino H, Kuroda S, Hirata K, Shiga T, Houkin K, Tamaki N. Predictors and clinical features of postoperative hyperperfusion after surgical revascularization for moyamoya disease: a serial single photon emission CT/positron emission tomography study. Stroke. 2012; 43: 2610-6.

24. Noguchi T, Kawashima M, Nishihara M, Egashira $Y$, Azama S, Irie H. Noninvasive method for mapping CVR in moyamoya disease using ASL-MRI. Eur J Radiol. 2015; 84: 1137-43.

25. Furuya K, Kawahara N, Morita A, Momose T, Aoki S, Kirino T. Focal hyperperfusion after superficial temporal artery-middle cerebral artery anastomosis in a patient with moyamoya disease. Case report. J Neurosurg. 2004; 100: 128-32.

26. Fujimura M, Tominaga T. Significance of Cerebral Blood Flow Analysis in the Acute Stage after Revascularization Surgery for Moyamoya Disease. Neurol Med Chir (Tokyo). 2015; 55: 775-81.

27. Hayashi T, Shirane R, Fujimura M, Tominaga T. Postoperative neurological deterioration in pediatric moyamoya disease: watershed shift and hyperperfusion. J Neurosurg Pediatr. 2010; 6: 73-81.

28. Fujimura $M$, Kaneta $T$, Tominaga $T$. Efficacy of superficial temporal artery-middle cerebral artery anastomosis with routine postoperative cerebral blood flow measurement during the acute stage in childhood moyamoya disease. Childs Nerv Syst. 2008; 24: 827-32.

29. Fujimura M, Kaneta T, Shimizu H, Tominaga T. Symptomatic hyperperfusion after superficial temporal artery-middle cerebral artery anastomosis in a child with moyamoya disease. Childs Nerv Syst. 2007; 23: 1195-8.

30. Zhao WG, Luo Q, Jia JB, Yu JL. Cerebral hyperperfusion syndrome after revascularization surgery in patients with moyamoya disease. Br J Neurosurg. 2013; 27: 321-5.

31. Ohkubo $\mathrm{K}$, Sakai $\mathrm{Y}$, Inoue $\mathrm{H}$, Akamine $\mathrm{S}$, Ishizaki $\mathrm{Y}$, Matsushita $\mathrm{Y}$, et al. Moyamoya disease susceptibility gene RNF213 links inflammatory and angiogenic signals in endothelial cells. Sci Rep. 2015; 5: 13191

32. Fujimura M, Gasche Y, Morita-Fujimura Y, Massengale J, Kawase M, Chan $\mathrm{PH}$. Early appearance of activated matrix metalloproteinase- 9 and blood-brain barrier disruption in mice after focal cerebral ischemia and reperfusion. Brain Res. 1999; 842: 92-100.
33. Hwang JW, Yang HM, Lee $\mathrm{H}$, Lee HK, Jeon YT, Kim JE, et al. Predictive factors of symptomatic cerebral hyperperfusion after superficial temporal artery-middle cerebral artery anastomosis in adult patients with moyamoya disease. Br J Anaesth. 2013; 110: 773-9.

34. Takahashi A, Kamiyama H, Houkin K, Abe H. Surgical treatment of childhood moyamoya disease--comparison of reconstructive surgery centered on the frontal region and the parietal region. Neurol Med Chir (Tokyo). 1995; 35: 231-7.

35. Wang Y, Chen L, Wang Y, Pan H, Wang Y, Xu B, et al. Hemodynamic study with duplex ultrasonography on combined (direct/indirect) revascularization in adult moyamoya disease. J Stroke Cerebrovasc Dis. 2014; 23 : 2573-9.

36. Zhang J, Wang J, Geng D, Li Y, Song D, Gu Y. Whole-brain CT perfusion and $\mathrm{CT}$ angiography assessment of Moyamoya disease before and after surgical revascularization: preliminary study with 256-slice CT. PLoS One. 2013; 8: e57595.

37. Uno M, Nakajima N, Nishi K, Shinno K, Nagahiro S. Hyperperfusion syndrome after extracranial-intracranial bypass in a patient with moyamoya disease--case report. Neurol Med Chir (Tokyo). 1998; 38: 420-4.

38. Hokari M, Kuroda S, Simoda Y, Uchino H, Hirata K, Shiga T, et al. Transient crossed cerebellar diaschisis due to cerebral hyperperfusion following surgical revascularization for moyamoya disease: case report. Neurol Med Chir (Tokyo). 2012; 52: 350-3.

39. Hayashi K, Horie N, Suyama K, Nagata I. Incidence and clinical features of symptomatic cerebral hyperperfusion syndrome after vascular reconstruction. World Neurosurg. 2012; 78: 447-54.

40. Takemoto Y, Morioka M, Nakagawa T, Hasegawa Y, Ohmori Y, Kawano T, et al. Prolonged and regionally progressive symptomatic cerebral hyperperfusion syndrome after superficial temporal artery-middle cerebral artery anastomosis in a patient with moyamoya disease. Surg Neurol Int. 2012; 3: 106 .

41. Ogasawara K, Komoribayashi N, Kobayashi M, Fukuda T, Inoue T, Yamadate $\mathrm{K}$, et al. Neural damage caused by cerebral hyperperfusion after arterial bypass surgery in a patient with moyamoya disease: case report. Neurosurgery. 2005; 56: E1380; discussion E.

42. Bacigaluppi S, Dehdashti AR, Agid R, Krings T, Tymianski M, Mikulis DJ. The contribution of imaging in diagnosis, preoperative assessment, and follow-up of moyamoya disease: a review. Neurosurg Focus. 2009; 26: E3.

43. Kohama M, Fujimura M, Mugikura S, Tominaga T. Temporal change of 3-T magnetic resonance imaging/angiography during symptomatic cerebral hyperperfusion following superficial temporal artery-middle cerebral artery anastomosis in a patient with adult-onset moyamoya disease. Neurosurg Rev. 2008; 31: 451-5; discussion 5.

44. Morisawa H, Kawamata T, Kawashima A, Hayashi M, Yamaguchi K, Yoneyama T, et al. Hemodynamics and changes after STA-MCA anastomosis in moyamoya disease and atherosclerotic cerebrovascular disease measured by micro-Doppler ultrasonography. Neurosurg Rev. 2013; 36: 411-9.

45. Han JH, Park YS, Lee WH, Koong SS, Min KS, Lee MS, et al. Cerebral-perfusion-based single-photon emission computed tomography (SPECT) staging using NeuroGam(R) in patients with moyamoya disease. Childs Nerv Syst. 2015

46. Tanioka S, Shiba M, Umeda Y, Sano T, Maeda M, Suzuki H. A Case of Moyamoya Disease with a Transient Neurologic Deterioration Associated with Subcortical Low Intensity on Fluid-Attenuated Inversion Recovery Magnetic Resonance Images After Bypass Surgery. World Neurosurg. 2015.

47. Chen $Y, X u$ W, Guo X, Shi Z, Sun Z, Gao L, et al. CT perfusion assessment of Moyamoya syndrome before and after direct revascularization (superficial temporal artery to middle cerebral artery bypass). Eur Radiol. 2016; 26: 254-61.

48. Fujimura $\mathrm{M}$, Niizuma $\mathrm{K}$, Endo $\mathrm{H}$, Sato $\mathrm{K}$, Inoue $\mathrm{T}$, Shimizu $\mathrm{H}$, et al. Quantitative analysis of early postoperative cerebral blood flow contributes to the prediction and diagnosis of cerebral hyperperfusion syndrome after revascularization surgery for moyamoya disease. Neurol Res. 2015; 37: 131-8.

49. Kawamata T, Kawashima A, Yamaguchi K, Hori T, Okada Y. Usefulness of intraoperative laser Doppler flowmetry and thermography to predict a risk of postoperative hyperperfusion after superficial temporal artery-middle cerebral artery bypass for moyamoya disease. Neurosurg Rev. 2011; 34: 355-62; discussion 62.

50. Nakagawa A, Fujimura M, Arafune T, Sakuma I, Tominaga T. Clinical implications of intraoperative infrared brain surface monitoring during superficial temporal artery-middle cerebral artery anastomosis in patients with moyamoya disease. J Neurosurg. 2009; 111: 1158-64.

51. Machida T, Ono J, Nomura R, Fujikawa A, Nagano O, Higuchi Y. Venous reddening as a possible sign of hyperperfusion after superficial temporal artery-middle cerebral artery anastomosis for moyamoya disease: case report. Neurol Med Chir (Tokyo). 2014; 54: 827-31.

52. Fujimura M, Inoue T, Shimizu H, Saito A, Mugikura S, Tominaga T. Efficacy of prophylactic blood pressure lowering according to a standardized postoperative management protocol to prevent symptomatic cerebral hyperperfusion after direct revascularization surgery for moyamoya disease. Cerebrovasc Dis. 2012; 33: 436-45.

53. Fujimura M, Shimizu H, Mugikura S, Tominaga T. Delayed intracerebral hemorrhage after superficial temporal artery-middle cerebral artery anastomosis in a patient with moyamoya disease: possible involvement of cerebral hyperperfusion and increased vascular permeability. Surg Neurol. 2009; 71: 223-7; discussion 7. 
54. Ohue S, Kumon Y, Kohno K, Watanabe H, Iwata S, Ohnishi T. Postoperative temporary neurological deficits in adults with moyamoya disease. Surg Neurol. 2008; 69: 281-6; discussion 6-7.

55. Fujimura $M$, Niizuma $K$, Inoue $T$, Sato $K$, Endo $H$, Shimizu $H$, et al Minocycline prevents focal neurological deterioration due to cerebral hyperperfusion after extracranial-intracranial bypass for moyamoya disease. Neurosurgery. 2014; 74: 163-70; discussion 70.

56. Januszewski J, Beecher JS, Chalif DJ, Dehdashti AR. Flow-based evaluation of cerebral revascularization using near-infrared indocyanine green videoangiography. Neurosurg Focus. 2014; 36: E14.

57. Kazumata K, Ito M, Tokairin K, Ito Y, Houkin K, Nakayama N, et al. The frequency of postoperative stroke in moyamoya disease following combined revascularization: a single-university series and systematic review. J Neurosurg. 2014; 121: 432-40.

58. Fujimura M, Kaneta T, Mugikura S, Shimizu H, Tominaga T. Temporary neurologic deterioration due to cerebral hyperperfusion after superficial temporal artery-middle cerebral artery anastomosis in patients with adult-onset moyamoya disease. Surg Neurol. 2007; 67: 273-82.

59. Schubert GA, Biermann P, Weiss C, Seiz M, Vajkoczy P, Schmiedek P, et al. Risk profile in extracranial/intracranial bypass surgery--the role of antiplatelet agents, disease pathology, and surgical technique in 168 direct revascularization procedures. World Neurosurg. 2014; 82: 672-7.

60. Ito A, Fujimura $\mathrm{M}$, Inoue $\mathrm{T}$, Tominaga $\mathrm{T}$. [Asymptomatic intracerebral hemorrhage under strict blood pressure control due to postoperative cerebral hyperperfusion in a patient with moyamoya disease]. No Shinkei Geka. 2011; 39: 681-6.

61. Mesiwala AH, Sviri G, Fatemi N, Britz GW, Newell DW. Long-term outcome of superficial temporal artery-middle cerebral artery bypass for patients with moyamoya disease in the US. Neurosurg Focus. 2008; 24: E15.

62. Ma Y, Li M, Jiao LQ, Zhang HQ, Ling F. Contralateral cerebral hemodynamic changes after unilateral direct revascularization in patients with moyamoya disease. Neurosurg Rev. 2011; 34: 347-53; discussion 53-4.

63. Esposito G, Fierstra J, Kronenburg A, Regli L. A comment on "Contralateral cerebral hemodynamic changes after unilateral direct revascularization in patients with moyamoya disease". Neurosurg Rev. 2012; 35: 141-3; author reply 3 .

64. Nho JS, Choi SE, Yi JW, Kang JM. Intraoperative acute brain swelling when performing indirect anastomosis in a patient with moyamoya disease -A case report. Korean J Anesthesiol. 2010; 59 Suppl: S191-3.

65. Jung YJ, Ahn JS, Kwon do H, Kwun BD. Ischemic complications occurring in the contralateral hemisphere after surgical treatment of adults with moyamoya disease. J Korean Neurosurg Soc. 2011; 50: 492-6.

66. Sim YW, Lee MS, Kim YG, Kim DH. Unpredictable postoperative global cerebral infarction in the patient of williams syndrome accompanying moyamoya disease. J Korean Neurosurg Soc. 2011; 50: 256-9.

67. Kuwabara Y, Ichiya Y, Sasaki M, Yoshida T, Masuda K, Matsushima T, et al. Response to hypercapnia in moyamoya disease. Cerebrovascular response to hypercapnia in pediatric and adult patients with moyamoya disease. Stroke. 1997; 28: 701-7.

68. Sato K, Shirane R, Yoshimoto T. Perioperative factors related to the development of ischemic complications in patients with moyamoya disease. Childs Nerv Syst. 1997; 13: 68-72.

69. Hyun SJ, Kim JS, Hong SC. Prognostic factors associated with perioperative ischemic complications in adult-onset moyamoya disease. Acta Neurochir (Wien). 2010; 152: 1181-8

70. Oshima H, Katayama Y, Hirayama T. Intracerebral steal phenomenon associated with global hyperemia in moyamoya disease during revascularization surgery. J Neurosurg. 2000; 92: 949-54.

71. Jagdevan S, Sriganesh K, Pandey P, Reddy M, Umamaheswara Rao GS. Anesthetic factors and outcome in children undergoing indirect revascularization procedure for moyamoya disease: An Indian perspective. Neurol India 2015 : 63: 702-6.

72. Antonucci MU, Burns TC, Pulling TM, Rosenberg J, Marks MP, Steinberg GK, et al. Acute Preoperative Infarcts and Poor Cerebrovascular Reserve Are Independent Risk Factors for Severe Ischemic Complications following Direct Extracranial-Intracranial Bypass for Moyamoya Disease. AJNR Am J Neuroradiol. 2015.

73. Kuroda S, Houkin K, Nunomura M, Abe H. Frontal lobe infarction due to hemodynamic change after surgical revascularization in moyamoya disease--two case reports. Neurol Med Chir (Tokyo). 2000; 40: 315-20.

74. Kuroda S, Hashimoto N, Yoshimoto T, Iwasaki Y, Research Committee on Moyamoya Disease in J. Radiological findings, clinical course, and outcome in asymptomatic moyamoya disease: results of multicenter survey in Japan. Stroke. 2007; 38: 1430-5.

75. Yamada S, Oki K, Itoh Y, Kuroda S, Houkin K, Tominaga T, et al. Effects of Surgery and Antiplatelet Therapy in Ten-Year Follow-Up from the Registry Study of Research Committee on Moyamoya Disease in Japan. J Stroke Cerebrovasc Dis. 2016; 25: 340-9.

76. Iwama T, Hashimoto N, Miyake H, Yonekawa Y. Direct revascularization to the anterior cerebral artery territory in patients with moyamoya disease: report of five cases. Neurosurgery. 1998; 42: 1157-61; discussion 61-2.

77. Freyschlag CF, Seiz M, Brockmann MA, Scharf J, Stier RW, Schubert GA, et al. Effect of mouth opening on bypass function after combined revascularization for Moyamoya disease. Acta Neurochir Suppl. 2011; 112: 35-8.
78. Amin-Hanjani $\mathrm{S}$, Singh A, Rifai $\mathrm{H}$, Thulborn KR, Alaraj A, Aletich $\mathrm{V}$, et al. Combined direct and indirect bypass for moyamoya: quantitative assessment of direct bypass flow over time. Neurosurgery. 2013; 73: 962-7; discussion 7-8.

79. Katsuta $\mathrm{T}$, Abe $\mathrm{H}$, Miki $\mathrm{K}$, Inoue $\mathrm{T}$. Reversible occlusion of donor vessel caused by mouth opening after superficial temporal artery-middle cerebral artery anastomosis in adult moyamoya patients. J Neurosurg. 2015; 123: 670-5.

80. Sekhar LN, Duff JM, Kalavakonda C, Olding M. Cerebral revascularization using radial artery grafts for the treatment of complex intracranial aneurysms: techniques and outcomes for 17 patients. Neurosurgery. 2001; 49: 646-58; discussion 58-9.

81. Natarajan SK, Hauck EF, Hopkins LN, Levy EI, Siddiqui AH. Endovascular management of symptomatic spasm of radial artery bypass graft: technical case report. Neurosurgery. 2010; 67: 794-8; discussion 8 .

82. Kuroda S, Houkin K. Bypass surgery for moyamoya disease: concept and essence of sugical techniques. Neurol Med Chir (Tokyo). 2012; 52: 287-94.

83. Aoki T, Yoshitomi M, Yamamoto M, Hirohata M, Morioka M. Ruptured de novo aneurysm arising at a site remote from the anastomosis 14 years after superficial temporal artery-middle cerebral artery bypass: a case report. Neurosurgery. 2012; 71: E905-9.

84. Nishimoto T, Yuki K, Sasaki T, Murakami T, Kodama Y, Kurisu K. A ruptured middle cerebral artery aneurysm originating from the site of anastomosis 20 years after extracranial-intracranial bypass for moyamoya disease: case report. Surg Neurol. 2005; 64: 261-5; discussion 5.

85. Eom KS, Kim DW, Kang SD. Intracerebral hemorrhage caused by rupture of a giant aneurysm complicating superficial temporal artery-middle cerebral artery anastomosis for moyamoya disease. Acta Neurochir (Wien). 2010; 152: 1069-73; discussion 73

86. Kawahara I, Morofuji $\mathrm{Y}$, Tsutsumi $\mathrm{K}$, Takahata $\mathrm{H}$, Ono $\mathrm{T}$, Toda $\mathrm{K}$, et al. De novo ruptured aneurysm at the site of anastomosis after superficial temporal artery-middle cerebral artery anastomosis--case report and literature review. Clin Neurol Neurosurg. 2013; 115: 457-60.

87. Zhang L, Xu K, Zhang Y, Wang X, Yu J. Treatment strategies for aneurysms associated with moyamoya disease. Int J Med Sci. 2015; 12: 234-42.

88. Mukerji N, Cook DJ, Steinberg GK. Is local hypoperfusion the reason for transient neurological deficits after STA-MCA bypass for moyamoya disease? J Neurosurg. 2015; 122: 90-4.

89. Horn P, Scharf J, Pena-Tapia P, Vajkoczy P. Risk of intraoperative ischemia due to temporary vessel occlusion during standard extracranial-intracranial arterial bypass surgery. J Neurosurg. 2008; 108: 464-9.

90. Sato H, Sato N, Tamaki N, Matsumoto S. Chronic low-perfusion state in children with moyamoya disease following revascularization. Childs Nerv Syst. 1990; 6: 166-71.

91. Takanari K, Araki Y, Okamoto S, Sato H, Yagi S, Toriyama K, et al. Operative wound-related complications after cranial revascularization surgeries. J Neurosurg. 2015: 1-6.

92. Houkin $\mathrm{K}$, Ishikawa $\mathrm{T}$, Yoshimoto $\mathrm{T}$, Abe $\mathrm{H}$. Direct and indirect revascularization for moyamoya disease surgical techniques and peri-operative complications. Clin Neurol Neurosurg. 1997; 99 Suppl 2: S142-5.

93. Kwon H, Kim HJ, Yim YM, Jung SN. Reconstruction of scalp defect after Moyamoya disease surgery using an occipital pedicle V-Y advancement flap. J Craniofac Surg. 2008; 19: 1075-9.

94. Jin SC, Oh CW, Kwon OK, Hwang G, Bang JS, Kang HS, et al. Epilepsy after bypass surgery in adult moyamoya disease. Neurosurgery. 2011; 68: 1227-32; discussion 32

95. Narisawa A, Fujimura $M$, Shimizu $H$, Tominaga $T$. [Seizure following superficial temporal-middle cerebral artery anastomosis in patients with moyamoya disease: possible contribution of postoperative cerebral hyperperfusion]. No Shinkei Geka. 2007; 35: 467-74.

96. Andoh T, Sakai N, Yamada H, Yano H, Hirayama H, Imao Y, et al. Chronic subdural hematoma following bypass surgery--report of three cases. Neurol Med Chir (Tokyo). 1992; 32: 684-9.

97. Sasamori T, Kuroda S, Nakayama N, Iwasaki Y. Incidence and pathogenesis of transient cheiro-oral syndrome after surgical revascularization for moyamoya disease. Neurosurgery. 2010; 67: 1054-9; discussion 60.

98. Feroze AH, Kushkuley J, Choudhri O, Heit JJ, Steinberg GK, Do HM Development of arteriovenous fistula after revascularization bypass for Moyamoya disease: case report. Neurosurgery. 2015; 11 Suppl 2: E202-6. 